\title{
SEARCH FOR IO, GANYMEDE AND EUROPA INDUCED RADIO EMISSIONS FROM CASSINI/RPWS INTEGRATED POWER TIME SERIES
}

\author{
L. Lamy*
}

\begin{abstract}
The Cassini mission flew by Jupiter in December 2000 and routinely observed Jupiter radio emissions with its Radio and Plasma Wave Science experiment (RPWS) over the interval 2000-2001, between a few $\mathrm{kHz}$ and $16 \mathrm{MHz}$, hence only missing the high frequency portion of decametric emissions. This database therefore provides an excellent basis to perform statistical studies, such as the search of Jupitersatellite interactions. Tracking individual structures such as 'arcs' in dynamic spectra recorded too close to Jupiter is difficult, owing to the large variety of observed emissions, and in turn prevents plotting their occurrence through usual drawings of the satellite phase as a function of the observer's longitude. Nonetheless, with long-term power time series, we can build similar plots directly using the intensity of the emission itself, once integrated over a chosen spectral range. This approach reveals clear signatures of emissions induced by Io, but also Ganymede and Europe which confirm their detection by other different techniques [see Louis et al., this issue; Zarka et al., this issue].
\end{abstract}

\footnotetext{
${ }^{*}$ LESIA, Observatoire de Paris, Meudon, France
} 
